Registered Report:

Conceptual fear generalization gradients

ACCEPTED IN-PRINCIPLE IN INTERNATIONAL JOURNAL OF PSYCHOPHYSIOLOGY

Gaëtan Mertens, Vera Bouwman, Arne Leer, and Iris M. Engelhard

Department of Clinical Psychology, Utrecht University, Utrecht, the Netherlands

Correspondence concerning this article should be addressed to Gaëtan Mertens, Department of Clinical Psychology, Heidelberglaan 1, room H1.29, Utrecht University, 3584CS Utrecht, the Netherlands.

E-mail: g.mertens@uu.nl

Tel: +31 302537553 


\begin{abstract}
A hallmark symptom of fear and anxiety disorder is generalization of fear to essentially innocuous stimuli and situations. Such generalization can occur through both perceptual and conceptually similarities. Recent studies indicate that perceptual generalization is inflated in anxiety patients and individuals prone to develop anxiety disorders, suggesting that perceptual generalization may be involved in the etiology of anxiety disorders. In the current study we want to address whether conceptual generalization is potentially implicated in the development of anxiety disorders. Therefore, we will use a novel paradigm in which the Dutch word mini [tiny] or enorm [enormous] is paired with an electric shock and assess fear to the conceptually related words klein [small], medium [medium], and groot [large]. The obtained generalization gradients will be related to personality traits known to be vulnerability factors for anxiety disorders. These results will provide insight into conceptual fear generalization and whether this phenomenon potentially relates to the development of anxiety disorders.
\end{abstract}

Keywords: Fear generalization; Conceptual generalization; Anxiety; Behavioral inhibition; Intolerance of uncertainty 


\section{Conceptual fear generalization gradients}

Anxiety disorders are characterized by not only fearing stimuli and situations that are dangerous or that closely resemble the context in which the original trauma occurred, but crucially also fearing stimuli and situations that are objectively safe or only faintly resemble the original trauma context. This 'overgeneralization' of fear causes great distress with anxiety disorder patients and often interferes with their daily tasks and routines. Hence, it has been suggested that overgeneralization may in fact be an etiological mechanism for the development of anxiety disorders (e.g., Lenaert et al., 2014; Lissek et al., 2014; Lissek \& Grillon, 2010).

Generalization of fear can be investigated in the laboratory by presenting participants with generalization stimuli (GSs) which resemble a stimulus (conditioned stimulus or $\mathrm{CS}+$ ) that was previously paired with an electric shock or another aversive stimulus (unconditioned stimulus or US). Typically it is observed that participants show a gradient of fear: they show more fear to GSs that more closely resemble the CS+ and gradually less fear to GSs that resemble the CS+ less closely (Lissek et al., 2008). Previous research has demonstrated that participants at risk for developing anxiety disorders (Lenaert et al., 2014; Wong \& Lovibond, 2018) and anxiety patients (Lissek et al., 2014, 2010) express more fear towards the GSs than control participants (i.e., overgeneralization of fear). These findings have been obtained using arbitrary stimuli (e.g., circles with varying diameters, dots on the screen with a varying location) and with different types of pathological anxiety (e.g., Generalized Anxiety Disorder, Post-Traumatic Stress Disorder), suggesting that overgeneralization may be a cross-diagnostic vulnerability factor for pathological anxiety (Lissek \& Grillon, 2010). However, it should also be mentioned that not all studies have replicated these findings (e.g., Lonsdorf \& Merz, 2017; Torrents-Rodas et al., 2012, 2013).

Most often, fear generalization is investigated with simple visual stimuli that vary on a perceptual dimension (i.e., circles of increasing size, see above). However, regardless of any perceptual similarity, fear can also generalize because stimuli are semantically related (e.g., doctors and needles), belong to the same category (e.g., honeybees and wasps), or vary along an abstract dimension (e.g., emotionality of faces) (Dymond, Dunsmoor, Vervliet, Roche, \& Hermans, 2015). Hence, rather than being perceptually similar, these different stimuli can be said to be conceptually similar, and fear may generalize through conceptual relatedness (Dunsmoor \& Murphy, 2015). 
It is likely that conceptual generalization is relatively more important than perceptual generalization in adult humans. That is, humans are highly trained and specialized in processing symbolic information (e.g., words, signs, digits). Symbols represent information regardless of the perceptual features of the represented objects. Many of the stimuli that adult humans daily encounter have symbolic meaning and their relatedness depends both on conceptual representations (e.g., prior knowledge, category membership, semantic networks) and perceptual features. Indeed, also in clinical features of anxiety disorders conceptual relatedness is most likely relevant. For instance, claustrophobics may fear planes, elevators, and overly crowded places, not because of their physical resemblance, but because all these situations involve a confined time and space in which no immediate escape is possible (see Radomsky, Rachman, Thordarson, McIsaac, \& Teachman, 2001). More generally, fear acquisition in real-world situations rarely involves simple sensory cues but rather consists of complex stimuli and situations with both perceptual features and symbolic meaning (e.g., a car accident with your children present, being humiliated during a presentation at work, losing a close friend due to a progressive disease). Therefore, fear generalization in humans likely involves conceptual generalization processes, and studying these processes may be essential to understand fear overgeneralization in anxiety disorders (Dunsmoor \& Murphy, 2015).

In the current research report, we provide a substantial research effort to investigate conceptual generalization in the laboratory and how this relates to risk factors for developing anxiety disorders. Therefore, we will introduce a novel paradigm to objectify conceptual generalization and investigate conceptual generalization in a sample of 120 individuals (see our sample size determination below) and correlate this with personality characteristics which are known risk factors for developing anxiety disorders. Particularly, we will present participants with related stimuli along a semantic dimension. Specifically, after fear conditioning with the word mini [tiny] or, counterbalanced, enorm [enormous], participants will be presented with the words klein [small], medium [medium], and groot [large]. This procedure will allow to calculate conceptual generalization gradients, which can provide additionally crucial variance to discriminate between participants at high and low risk to develop anxiety disorders (see Lenaert et al., 2014; Lissek \& Grillon, 2010). If our paradigm proves useful for discrimination, it could potentially also be 
used for the screening, prevention and treatment (e.g., using discrimination training; Dunsmoor \& LaBar, 2013) of anxiety disorders.

Fear will be operationalized, as is common in fear conditioning research, with physiological responses (skin conductance responses and fear potentiated startle) and subjective ratings (US expectancy). There is some debate in the literature about which measures most closely correspond with fear (and about what fear exactly is; Fanselow \& Pennington, 2018; LeDoux \& Pine, 2016). Our view is that fear constitutes an integrated response of subjective apprehensions, physiological responses and action tendencies. Though the correspondence between these components is not perfect, we see them, particularly in the strong situation of a fear conditioning experiment, as interchangeable indices of fear. Accordingly, we will correct for multiple testing between these different measures of fear. As measures of individual differences in the risk of developing anxiety disorders we included trait anxiety, behavioral inhibition, and intolerance of uncertainty. These personality traits are related to the development of anxiety disorders (Carver \& White, 1994; Clark, Watson, \& Mineka, 1994; Clauss \& Blackford, 2012; Lonsdorf \& Merz, 2017; McEvoy \& Mahoney, 2012; Spielberger, Gorsuch, Lushene, Vagg, \& Jacobs, 1983). Though these constructs partly overlap (i.e., they have positive non-zero correlations with each other; see for instance Sjouwerman, Scharfenort, \& Lonsdorf, 2018), they have been distinguished from each other in the literature (for a recent review see Lonsdorf \& Merz, 2017). At present, it is unclear which of these personality traits would more closely be related to overgeneralization. As such, we do not have a specific focal hypothesis of which personality trait would correlate most strongly with fear generalization.

In summary, our hypotheses are the following:

(1) During acquisition we predict to find larger fear responses (i.e., expectancy ratings, skin conductance responses, and fear potentiated startle) to the CS+ compared to the CS-.

(2) During generalization we expect to find increasingly larger fear responses to the CS-, the GSs, and the CS+ (i.e., conceptual generalization).

(3) We predict that smaller differences in fear responses between the CS+ and the GSs (i.e., more conceptual overgeneralization) will be related to anxious personality traits, similar to what has previously been observed for perceptual generalization (Lenaert et al., 2014; Lissek et al., 2008; Morriss, Macdonald, \& van Reekum, 2016; Wong \& Lovibond, 2018). 
(4) We do not have specific hypotheses about which fear responses will be most sensitive to conceptual fear overgeneralization. Because we believe that the different fear measures operationalize the same construct, we will correct for multiple testing when calculating the correlations between the different fear measures and the personality questionnaires.

(5) We do not have specific hypotheses about which personality trait will be most sensitive to fear generalization. The different personality traits have been distinguished in the literature, but it is unclear which trait most closely relates to fear overgeneralization. Therefore, this aspect of our study is exploratory. Significant correlations between conceptual fear overgeneralization and a specific personality questionnaire will be followed up with additional multiple regression analyses to establish the specificity of the correlations over the shared variance with the other personality questionnaires (see below).

\section{Method}

\section{Power analysis}

An a priori power calculation was performed using $G^{*}$ power (Faul, Erdfelder, Lang, \& Buchner, 2007) to determine the required sample size for the crucial correlations between the amount of conceptual fear generalization and individuals differences in anxiety sensitivity. The alpha cut-off criteria was set at .017 (i.e., .05 divided by 3 ) to correct for multiple testing (i.e., three different fear measures to assess generalization). The power analysis indicated a required sample size of 120 to detect a medium effect size $(r=0.3)$ with a power of .90 .

\section{Participants}

120 participants (see above), aged between 18 and 35 years, will participate in the study. Participants will be female or male, mostly undergraduate students, recruited at Utrecht University. Inclusion criteria will be Dutch as first language; good (corrected) hearing and vision; no medication use which can impair attention, reaction time, memory, or concentration; no psychiatric disorder in the last two years; not being under treatment with a psychiatrist or psychologist currently or in the past two years; no pregnancy; and no current or past serious neurological or medical conditions (such as epilepsy or heart disease). Participants will provide informed consent and will receive money ( 8 euros per hour) or course credits as incentives. 
Participants who failed the contingency test, the conceptual gradient test (see the Data Exclusion section below), or were excluded for any other reason (e.g., psychophysiological data quality; see below) will be replaced by other participants to maintain our targeted sample size for the final analyses.

\section{Design}

The current study design is based on the generalization paradigm of Lissek et al. (2008). Instead of using different sizes of rings as stimuli, this study will use words that differentially refer to 'size' (see Table 1). The experiment consists of three phases: (1) Practice phase; (2) Acquisition phase; and (3) Generalization phase. In each phase, participants will be exposed to two stimuli (i.e., the CS+ and CS-). In the generalization phase, participants will also see generalization stimuli (see below). The different phases will follow each other consecutively within the same test session. The design of the experiment is completely within-subjects, except for the betweensubjects factor 'counterbalancing'. See Figure 1 for a schematic overview of the experiment.

$\begin{array}{lccc}\begin{array}{c}\text { Practice } \\ \text { Phase }\end{array} & \begin{array}{c}\text { Acquisition } \\ \text { Phase }\end{array} & \begin{array}{c}\text { Generalization } \\ \text { Phase }\end{array} \\ \text { CS+ } & \text { Mini } & \text { Mini } & \text { Mini } \\ \text { GS1 } & & & \text { Medium } \\ \text { GS3 } & & & \text { Groot } \\ \text { CS- } & \text { Enorm } & \text { Enorm } & \text { Enorm }\end{array}$

Figure 1. Schematic overview of the design of the experiment. Allocation of the words mini [tiny] and enorm [enormous] was counterbalanced over participants. Note: CS $-=$ conditioned stimulus never paired with shock; $\mathrm{CS}+=$ conditioned stimulus occasionally paired with shock; GS = generalization stimulus. 


\section{Materials}

Stimuli. Five words related to the concept size will be used as conditioned stimuli (CS+ and CS-) and generalization stimuli (GSs, see Figure 1). The most extreme words will serve as CS+ and CS-. Whether the word enorm or mini serves as CS+ will be counterbalanced across participants. These stimuli will be presented on a 21 inch computer screen (HP EliteDisplay E231) with a screen resolution of 1920 by 1080 pixels. The words will be displayed in the middle of the screen with font style 'Arial' and font size 36.

Questionnaires. We will use three commonly used questionnaires that measure personality traits that are thought to constitute risk factors for the development of anxiety disorders (Lonsdorf \& Merz, 2017). Particularly, the StateTrait Anxiety Inventory, trait version (STAI-DY-2; Spielberger et al., 1983; Dutch version: van der Ploeg, Defares, \& Spielberger, 2000) will be used to assess trait anxiety levels. The STAI consists of 20 items measuring state anxiety (STAI-S) and 20 items measuring trait anxiety (STAI-T). Responses are given on a 4-point Likert scale $(1=$ Not at all/Almost never, $4=$ Very much so/Almost always $)$. Furthermore, the intolerance of Uncertainty Scale (IUS, Dutch translation: de Bruin, Rassin, van der Heiden, \& Muris, 2006; original French version: Freeston, Rhéaume, Letarte, Dugas, \& Ladouceur, 1994) will be used to assess emotional, cognitive, and behavioral reactions to ambiguous situations, implications of being uncertain, and attempts to control the future. The scale consists of 27 items that can be answered on a 5-point Likert $(1=$ Not at all characteristic of me, $5=$ Entirely characteristic of me $)$. Finally, behavioral inhibition will be assessed with the behavioral inhibition subscale of the BIS/BAS Scales questionnaire (Carver \& White, 1994; Dutch translation: Franken, Muris, \& Rassin, 2005). This questionnaire consists of 24 questions that can be answered with four response options $(1=$ strong agreement, $2=$ slight agreement, $3=$ slight disagreement, $4=$ strong disagreement), of which seven items constitute the behavioral inhibition subscale.

Physiological apparatus. A $500 \mathrm{~ms}$ electric shock delivered to the dominant hand will serve as US, administered using a Digitimer DS7A device (see https://digitimer.com/). Participants can determine the level of the shock such that it is not painful but will be highly uncomfortable (Mertens \& De Houwer, 2016).

Skin conductance response (SCR) will be measured using two BioSemi GSR electrodes (BioSemi, Amsterdam, the Netherlands) that will be filled with conductive 
gel and will be attached on the non-dominant hand of the participant. Startle response will be measured using four BioSemi FLAT active electrodes that will be filled with conductive gel. Two electrodes will be attached under the left eye (orbicularis oculi muscle) and two on the forehead as ground electrodes. Startle will be elicited by a 95 $\mathrm{dB}$ white noise probe for $50 \mathrm{~ms}$.

US expectancy. The extent to which participants expect the US during a CS or GS will be measured using an online (i.e., during CS/GS presentation) 9-point Likert scale (1 = Definitely no shock, 5 = Uncertain, 9 = Definitely a shock). The US expectancy scale will be presented on the bottom of the screen. Participants have to click on a number with the mouse to lock their answer. They can only lock their answer once.

\section{Procedure}

The conditioning and generalization task are programmed and presented using Inquisit (v4). Participants will take place in a dimmed and soundproof room, $60 \mathrm{~cm}$ away from the computer screen. First, shock electrodes will be attached and a shock workup procedure will be completed (see Mertens \& De Houwer, 2016). Electrodes measuring startle response and skin conductance, and headphones will be attached. Participants will start by filling in the questionnaires. Hereafter, participants will start with the Practice phase consisting of three trials consisting of random words to practice filling in the US expectancy Likert scale. No US will be presented in this phase. Following the Practice phase, participants will be presented nine times with the startle probe with an inter-probe interval of 19, 21, or $23 \mathrm{~s}$. After startle habituation, the experiment immediately starts with the Acquisition phase. CSs will be presented for $8 \mathrm{~s}$ in the middle of the computer screen. During CS presentation, participants will fill in the US expectancy Likert scale. At 7 s post-CS onset a startle probe will be presented. Six of the eight CS+ trials will immediately be followed by the US (75\% reinforcement schedule), while the CS- will never be followed by the US. During an ITI trial, a startle probe will be presented without the presentation of a stimulus. Interprobe interval will be maintained at 19, 21, or 23 s throughout the experiment. At the end of the Acquisition phase, participants will be asked how anxious they feel about the CS+ and CS- $(1=$ Not anxious, $100=$ Very anxious $)$. The Generalization phase starts after a 10-min break. During the Generalization phase, all CSs and GSs will also be presented for $8 \mathrm{~s}$ and after $7 \mathrm{~s}$ the startle probe is presented. Four out of eight CS+ trials will immediately be followed by the US (50\% reinforcement schedule; see 
Lissek et al., 2008). The experiment will end with a manipulation check. To check whether participants interpreted the words as intended, they will be asked to list words in increasing order. Furthermore, participants will fill in four retrospective questions: (1) Which word predicted the electric shock?; (2) How certain are you about your answer? $(1$ = completely certain, $2=$ fairly certain, $3=$ fairly uncertain, $4=$ completely uncertain); (3) How anxious do you feel about the word 'Enormous'? (1 = Not anxious, $100=$ Very anxious); and (4) How anxious do you feel about the word 'Mini'[/ 'Enorm']? (1 = Not anxious, $100=$ Very anxious $)$. Participants will be debriefed and incentives will be given.

\section{Proposed data-analysis pipeline}

\section{Preprocessing steps}

Data exclusions. Participants who did not learn the contingency, will be excluded from the analyses. This will be checked by looking at the contingency question. Participants must correctly state which word is followed by the electric shock and have to indicate that they are completely certain or fairly certain about their answer (Singh, Dawson, Schell, Courtney, \& Payne, 2013).

Furthermore, we will check whether participants perceived the gradient of the words used in the experiment as we intended. If they list the word in any other order than: mini, klein, medium, groot, and enorm, their data will be excluded from the analyses.

Finally, participants will be excluded based on insufficient psychophysiological data quality. Psychophysiological data quality will first be assessed based on visual inspection of the signal: Completely flat lines or highly noisy data usually indicate the disconnection of electrodes. The data from participants showing such artefacts will be excluded. Additionally, data will be excluded if participants do not show any discernible SCRs towards the US administration (no responses $>0.02 \mu \mathrm{S}$; see below) or more than $50 \%$ unusable startle response datapoints $(\mu \mathrm{V}$ maximum peak in the response window $<$ average $\mu \mathrm{V}$ baseline; see below).

Excluded participants will be replaced by new participants to maintain the targeted sample size $(N=120)$. We will not exclude any participants based on their performance on the outcome measures (e.g., successful fear acquisition on SCRs/FPS/US expectancy ratings) because this can potentially lead to sample 
selection effects, which can attenuate correlations with inter-individual differences (Lonsdorf et al., 2017).

Generalization index (GI). A generalization index for each participant will be calculated during the Generalization phase. For each participant, the average fear responses towards the three GSs will be calculated and will be divided by the fear responses towards the CS+ (separately for US expectancy, startle responses and SCRs). This formula corrects for individual differences in initial response strength (Leer, Sevenster, \& Lommen, 2018; Lenaert, van de Ven, Kaas, \& Vlaeyen, 2016). Higher GI scores represent more generalization (i.e., '1' reflects full generalization; ' 0 ' reflects no generalization). This index can be used to correlate the amount of conceptual fear generalization to the trait anxiety, IU, and behavioral inhibition scores of participants.

Skin conductance response. The skin conductance signal will first be downsampled to $10 \mathrm{~Hz}$ using BrainVision Analyzer software (Brain Products, Munich, Germany). Responses will be computed by subtracting the mean skin conductance response for the $2 \mathrm{~s}$ preceding CS onset from the highest value recorded during the full 7 s CS-US interval (Pineles, Orr, \& Orr, 2009). A response threshold of $0.02 \mu \mathrm{S}$ will be applied for the SCRs (Boucsein et al., 2012). Responses below this cut-off will be replaced with 0 . Each participant's SCR score will be divided by its maximum response to minimize inter-individual variance (Boucsein et al., 2012). A square-root transformation will be applied to normalize the distribution (Dawson, Schell, Filion, \& Berntson, 2007).

Startle response. The electromyographic signal will be filtered $(28-500 \mathrm{~Hz})$, rectified, and smoothed (15.9 Hz low-pass filter) using BrainVision Analyzer. Response amplitudes will be computed as the difference between the maximum startle response within 21 to $150 \mathrm{~ms}$ after stimulus onset and the average startle response during baseline (-30 to $20 \mathrm{~ms}$ after stimulus onset). An individual range correction (a T-transformation) will be applied by standardizing each blink amplitude using all scores for a given subject as the reference distribution (Blumenthal et al., 2005).

\section{Precise description of all planned analyses}

Acquisition. A repeated measures ANOVA with within-subject factors Stimulus (CS+ vs CS-) and Trial (1 to 8) will be performed on US expectancy ratings, startle responses, and SCRs. We expect a significant main effect of Stimulus and an interaction between Stimulus and Trial for all the different measures, indicating 
successful fear acquisition. Paired $t$-tests will be performed on the first and last CS+ and CS- trials for all outcome measures to verify fear acquisition.

Generalization test. A repeated measures ANOVA with factor Stimulus (CS+, CS-, GS1, GS2, GS3) and Counterbalancing (mini or enorm as CS+) will be performed on US expectancy ratings, startle responses, and SCRs. Only a main effect of Stimulus is expected for all outcome measures. Follow-up paired sample t-tests will be performed to compare fear responses to CS- with GS1, GS2, GS3, and CS+.

Correlational analyses. The correlation between the GI and scores on the three questionnaires will be assessed using correlation analyses (Pearson or Spearman, depending on the characteristics of distributions of the variables at hand).

Specificity analyses. Significant correlations between GI and the questionnaires will be followed by tests for specificity. Particularly, multiple regression analyses will be conducted to investigate whether the relationship between GI and one of the questionnaires remains significant when controlling for the shared variance with the other questionnaires.

\section{Timeline}

Upon acceptance of our study plan, data collection can commence immediately given that the required materials are already available (see https://osf.io/k36ba/) and our planned sample consists of students. Based on experience with similar previous studies, we estimate that testing 120 participants in the lab will take approximately three months of testing. Data analyses and writing up the results will require approximately one additional month. 


\section{References}

Blumenthal, T. D., Cuthbert, B. N., Filion, D. L., Hackley, S., Lipp, O. V., \& Van Boxtel, A. (2005). Committee report: Guidelines for human startle eyeblink electromyographic studies. Psychophysiology, 42(1), 1-15. https://doi.org/10.1111/j.1469-8986.2005.00271.x

Boucsein, W., Fowles, D. C., Grimnes, S., Ben-Shakhar, G., Roth, W. T., Dawson, M. E., \& Filion, D. L. (2012). Publication recommendations for electrodermal measurements. Psychophysiology, 49(8), 1017-1034. https://doi.org/10.1111/j.1469-8986.2012.01384.x

Carver, C. S., \& White, T. L. (1994). Behavioral inhibition, behavioral activation, and affective responses to impending reward and punishment: The BIS/BAS Scales. Journal of Personality and Social Psychology, 67(2), 319-333. https://doi.org/10.1037/0022-3514.67.2.319

Clark, L. A., Watson, D., \& Mineka, S. (1994). Temperament, personality, and the mood and anxiety disorders. Journal of Abnormal Psychology, 103(1), 103-116.

Clauss, J. A., \& Blackford, J. U. (2012). Behavioral Inhibition and Risk for Developing Social Anxiety Disorder: A Meta-Analytic Study. Journal of the American Academy of Child \& Adolescent Psychiatry, 51(10), 1066-1075.e1. https://doi.org/10.1016/j.jaac.2012.08.002

Dawson, M. E., Schell, A. M., Filion, D. L., \& Berntson, G. G. (2007). The Electrodermal System. In J. T. Cacioppo, L. G. Tassinary, \& G. Berntson (Eds.), Handbook of Psychophysiology (3rd ed., pp. 157-181). Cambridge: Cambridge University Press. https://doi.org/10.1017/CBO9780511546396

de Bruin, G. O., Rassin, E., van der Heiden, C., \& Muris, P. (2006). Psychometric properties of a Dutch version of the Intolerance of Uncertainty Scale.

Netherlands Journal of Psychology, 62(2), 87-92. https://doi.org/10.1007/BF03061055

Dunsmoor, J. E., \& LaBar, K. S. (2013). Effects of discrimination training on fear generalization gradients and perceptual classification in humans. Behavioral Neuroscience, 127(3), 350-356. https://doi.org/10.1037/a0031933

Dunsmoor, J. E., \& Murphy, G. L. (2015). Categories, concepts, and conditioning: how humans generalize fear. Trends in Cognitive Sciences, 19(2), 73-77. https://doi.org/10.1016/j.tics.2014.12.003

Dymond, S., Dunsmoor, J. E., Vervliet, B., Roche, B., \& Hermans, D. (2015). Fear 
Generalization in Humans: Systematic Review and Implications for Anxiety Disorder Research. Behavior Therapy, 46(5), 561-582. https://doi.org/10.1016/j.beth.2014.10.001

Fanselow, M. S., \& Pennington, Z. T. (2018). A return to the psychiatric dark ages with a two-system framework for fear. Behaviour Research and Therapy, 100(August 2017), 24-29. https://doi.org/10.1016/j.brat.2017.10.012

Faul, F., Erdfelder, E., Lang, A.-G., \& Buchner, A. (2007). G*Power 3: A flexible statistical power analysis program for the social, behavioral, and biomedical sciences. Behavior Research Methods, 39(2), 175-191. https://doi.org/10.3758/BF03193146

Franken, I. H. A., Muris, P., \& Rassin, E. (2005). Psychometric Properties of the Dutch BIS/BAS Scales. Journal of Psychopathology and Behavioral Assessment, 27(1), 25-30. https://doi.org/10.1007/s10862-005-3262-2

Freeston, M. H., Rhéaume, J., Letarte, H., Dugas, M. J., \& Ladouceur, R. (1994). Why do people worry? Personality and Individual Differences, 17(6), 791-802. https://doi.org/10.1016/0191-8869(94)90048-5

LeDoux, J. E., \& Pine, D. S. (2016). Using Neuroscience to Help Understand Fear and Anxiety: A Two-System Framework. American Journal of Psychiatry, 173(11), 1083-1093. https://doi.org/10.1176/appi.ajp.2016.16030353

Leer, A., Sevenster, D., \& Lommen, M. J. J. (2018). Generalisation of threat expectancy increases with time. Cognition and Emotion, 0(0), 1-9. https://doi.org/10.1080/02699931.2018.1526167

Lenaert, B., Boddez, Y., Griffith, J. W., Vervliet, B., Schruers, K., \& Hermans, D. (2014). Aversive learning and generalization predict subclinical levels of anxiety: A six-month longitudinal study. Journal of Anxiety Disorders, 28(8), 747-753. https://doi.org/10.1016/j.janxdis.2014.09.006

Lenaert, B., van de Ven, V., Kaas, A. L., \& Vlaeyen, J. W. S. (2016). Generalization on the Basis of Prior Experience Is Predicted by Individual Differences in Working Memory. Behavior Therapy, 47(1), 130-140. https://doi.org/10.1016/j.beth.2015.10.001

Lissek, S., Biggs, A. L., Rabin, S. J., Cornwell, B. R., Alvarez, R. P., Pine, D. S., \& Grillon, C. (2008). Generalization of conditioned fear-potentiated startle in humans: experimental validation and clinical relevance. Behaviour Research and Therapy, 46(5), 678-687. https://doi.org/10.1016/j.brat.2008.02.005 
Lissek, S., Bradford, D. E., Alvarez, R. P., Burton, P., Espensen-Sturges, T., Reynolds, R. C., \& Grillon, C. (2013). Neural substrates of classically conditioned fear-generalization in humans: a parametric fMRI study. Social Cognitive and Affective Neuroscience. https://doi.org/10.1093/scan/nst096

Lissek, S., \& Grillon, C. (2010). Overgeneralization of conditioned fear in the anxiety disorders: Putative memorial mechanisms. Zeitschrift Für Psychologie/Journal of Psychology, 218, 146-148. https://doi.org/https://doi.org/10.1027/0044$3409 / \mathrm{a} 000022$

Lissek, S., Kaczkurkin, A. N., Rabin, S., Geraci, M., Pine, D. S., \& Grillon, C. (2014). Generalized Anxiety Disorder Is Associated With Overgeneralization of Classically Conditioned Fear. Biological Psychiatry, 75(11), 909-915. https://doi.org/10.1016/j.biopsych.2013.07.025

Lissek, S., Rabin, S., Heller, R. E., Lukenbaugh, D., Geraci, M., Pine, D. S., \& Grillon, C. (2010). Overgeneralization of Conditioned Fear as a Pathogenic Marker of Panic Disorder. American Journal of Psychiatry, 167(1), 47-55. https://doi.org/10.1176/appi.ajp.2009.09030410

Lonsdorf, T. B., Menz, M. M., Andreatta, M., Fullana, M. A., Golkar, A., Haaker, J., ... Merz, C. J. (2017). Don't fear 'fear conditioning': Methodological considerations for the design and analysis of studies on human fear acquisition, extinction, and return of fear. Neuroscience \& Biobehavioral Reviews. https://doi.org/10.1016/j.neubiorev.2017.02.026

Lonsdorf, T. B., \& Merz, C. J. (2017). More than just noise: Inter-individual differences in fear acquisition, extinction and return of fear in humans Biological, experiential, temperamental factors, and methodological pitfalls. Neuroscience and Biobehavioral Reviews, 80(June), 703-728. https://doi.org/10.1016/j.neubiorev.2017.07.007

McEvoy, P. M., \& Mahoney, A. E. J. (2012). To Be Sure, To Be Sure: Intolerance of Uncertainty Mediates Symptoms of Various Anxiety Disorders and Depression. Behavior Therapy, 43(3), 533-545. https://doi.org/10.1016/j.beth.2011.02.007

Mertens, G., \& De Houwer, J. (2016). Potentiation of the startle reflex is in line with contingency reversal instructions rather than the conditioning history. Biological Psychology, 113, 91-99. https://doi.org/10.1016/j.biopsycho.2015.11.014

Morriss, J., Macdonald, B., \& van Reekum, C. M. (2016). What Is Going On Around Here? Intolerance of Uncertainty Predicts Threat Generalization. PLOS ONE, 
11(5), e0154494. https://doi.org/10.1371/journal.pone.0154494

Pineles, S. L., Orr, M. R., \& Orr, S. P. (2009). An alternative scoring method for skin conductance responding in a differential fear conditioning paradigm with a longduration conditioned stimulus. Psychophysiology, 46(5), 984-995. https://doi.org/10.1111/j.1469-8986.2009.00852.x

Radomsky, A. S., Rachman, S., Thordarson, D. S., McIsaac, H. K., \& Teachman, B. A. (2001). The Claustrophobia Questionnaire. Journal of Anxiety Disorders, 15(4), 287-297. https://doi.org/10.1016/S0887-6185(01)00064-0

Singh, K., Dawson, M. E., Schell, A. M., Courtney, C. G., \& Payne, A. F. H. (2013). Can human autonomic classical conditioning occur without contingency awareness? The critical importance of the trial sequence. Biological Psychology, 93(1), 197-205. https://doi.org/10.1016/j.biopsycho.2013.02.007

Sjouwerman, R., Scharfenort, R., \& Lonsdorf, T. B. (2018). Individual differences in fear learning: Specificity to trait-anxiety beyond other measures of negative affect, and mediation via amygdala activation. BioRxiv, 1-22. https://doi.org/10.1101/233528

Spielberger, C. D., Gorsuch, R. L., Lushene, R., Vagg, P. R., \& Jacobs, G. A. (1983). Manual for the State-Trait Anxiety Inventory. Palo Alto, CA: Consulting Psychologists Press.

Torrents-Rodas, D., Fullana, M. A., Arias, B., Bonillo, A., Caseras, X., Andión, O., ... Torrubia, R. (2012). Acquisition and generalization of fear conditioning are not modulated by the BDNF-val66met polymorphism in humans. Psychophysiology, 49(5), 713-719. https://doi.org/10.1111/j.14698986.2011.01352.x

Torrents-Rodas, D., Fullana, M. a, Bonillo, A., Caseras, X., Andión, O., \& Torrubia, R. (2013). No effect of trait anxiety on differential fear conditioning or fear generalization. Biological Psychology, 92(2), 185-190. https://doi.org/10.1016/j.biopsycho.2012.10.006

van der Ploeg, H. M., Defares, P. B., \& Spielberger, C. D. (2000). Handleiding bij de Zelfbeoordelings Vragenlijst. Een Nederlandstalige bewerking van de Spielberger State-Trait Anxiety Inventory. Lisse, The Netherlands.

Wong, A. H. K., \& Lovibond, P. F. (2018). Excessive generalisation of conditioned fear in trait anxious individuals under ambiguity. Behaviour Research and Therapy, 107(January), 53-63. https://doi.org/10.1016/j.brat.2018.05.012 
